\title{
The Design of Optimal PID Control Method for Quadcopter Movement Control
}

\author{
Hanum Arrosida a,1,*, Mohammad Erik Echsony ${ }^{\mathrm{b}, 2}$ \\ Politeknik Negeri Madiun, Jl.Serayu No.84, Madiun 63133, Indonesia \\ hanumarrosida@pnm.ac.id,erik_sony@pnm.ac.id
}

Article history:

Received: 13-11-2017

Revised: 29-12-2017

Accepted: 11-02-2018

Keywords:

Quadcopter

PID Optimal

Tuning Gain

Fungsi Energi Minimum

\begin{abstract}
Nowadays, quadcopter motion control has become a popular research topic because of its versatile ability as an unmanned aircraft can be used to alleviate human labor and also be able to reach dangerous areas or areas which is unreachable to humans. On the other hand, the Optimal PID control method, which incorporates PID and Linear Quadratic Regulator (LQR) control methods, has also been widely used in industry and research field because it has advantages that are easy to operate, easy design, and a good level of precision. In the PID control method, the main problem to be solved is the accuracy of the gain value $\mathrm{Kp}, \mathrm{Ki}$, and $\mathrm{Kd}$ because the inappropriateness of those value will result in an imprecise control action. Based on these problems and referring to the previous study, the optimal PID control method was developed by using PID controller structure with tuning gain parameter of PID through Linear Quadratic Regulator (LQR) method. Through the integration of these two control methods, the optimum solutions can be obtained: easier controller design process for quadcopter control when crossing the determined trajectories, steady state error values less than 5\% and a stable quadcopter movement with roll and pitch angle stabilization at position 0 radians with minimum energy function.
\end{abstract}

Copyright $\odot 2017$ International Journal of Artificial Intelegence Research. All rights reserved.

\section{Introduction}

It is a challenge to control quadcopter automatically, because quadcopter has high maneuverability, high nonlinearity, and has six degrees of freedom with four actuators [1]. In recent years, various control methods have been explored and applied for position control and quadcopter orientation [2]. Conventional control methods such as PID are widely used for quadcopter motion control with the linear model approach, but the nonlinearity effects of quadcopter cannot be well compensated by PID controllers if the parameter gain is used incorrectly [3]. Based on Implementation of conventional PID controllers on nonlinear systems with significant delay requires a longer time to achieve a stable state. To overcome this problem, it is necessary to implement a combination of PID-based controller and fuzzy logic that function as tuning parameter values [4].

In addition to tuning the constants of $\mathrm{P}, \mathrm{I}$ and $\mathrm{D}$, proper determination of setpoint errors is also required to make the robot's response become faster so that when it gets interrupted, the robot can return to the steady state and can overcome the interference that occurred [5]. In the previous research, the motion control applied to quadcopter was linear quadratic regulator (LQR) control method by minimizing the energy function to obtain the weighted value of $\mathrm{Q}$ matrix for optimal result [6]. This weighted Q matrix can be used for tuning gain of PID controller parameters so that the applied PID controller can produce an optimal response. However In this research, a method of optimal PID control with automatic tuning gain through LQR (Linear Quadratic Regulator) method is proposed [7]. The advantage of combining these two control methods is an easier control design with optimal results, so it can be used appropriately for motion control on the quadcopter [8]. 


\section{Proposed Method}

Linear Quadratic Regulator is an optimal control on a linear system with a quadratic criterion to solve regulator problem [9].

The general formula of state equation is as follow:

$$
\begin{aligned}
& x=A x+B u \\
& y=C x
\end{aligned}
$$

with,

$$
\begin{array}{ll}
x_{n^{* 1}} & : \text { System State } \\
u_{m^{*} n}: \text { Input State } \\
y_{1^{* 1}}: \text { Output State } \\
A \quad: \text { System Matrix } A_{n^{*} n} \\
B \quad: \text { System Matrix } B_{n^{*} m} \\
C \quad: \text { System Matrix } C_{1^{*} n}
\end{array}
$$

The equation for minimizing the energy (cost function/quadratic function) through performance index of the interval $\left[t_{0}, \infty\right]_{\left[t_{0}, \infty\right]}$ is as follow:

$$
J=\frac{1}{2} \int_{t_{0}}^{\infty}\left(e^{T} Q e+u^{T} R u\right) d t
$$

with,

$t_{0}=$ starting time

$\infty=$ end time

$Q=$ positive semi-definite matrix

$R=$ positive definite matrix

On the regulator problem can be solved by finishing Riccati Equation on the following equation:

$$
A^{T} P+P A-P B R^{-1} B^{T} P+Q=0
$$

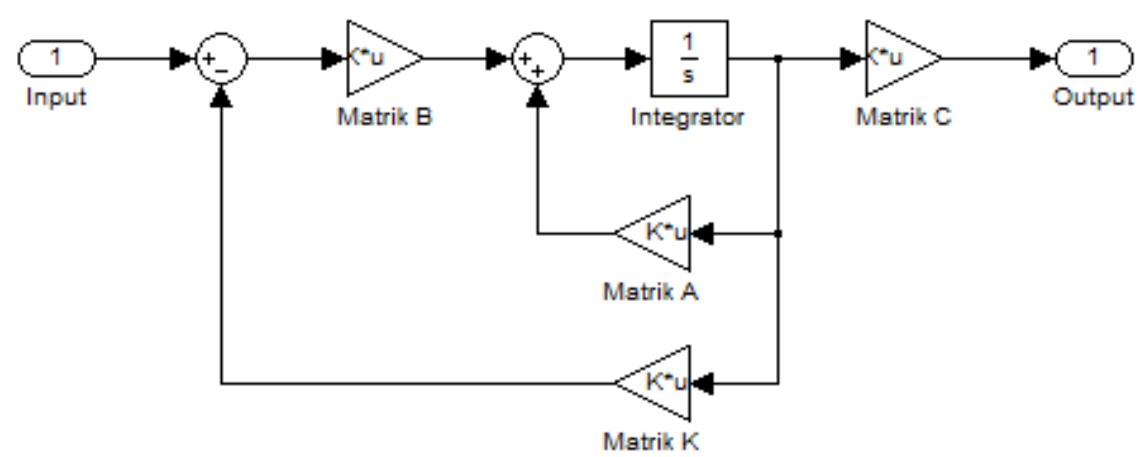

Fig. 1.Optimal Control Block Diagram 
For the LQR controller design, its selection of weighted matrix $\mathrm{Q}$ and $\mathrm{R}$ are based on [10] and [11]. The greater the value of $\mathrm{Q}$, the closer to the minimum. The greater the value of $\mathrm{R}$, the smaller / minimum energy used. Fig 1 shows the block diagram of the optimal control. The value of the Riccati Equation solution is attempted to be a small value matrix, namely Optimal PID Controller [12].

$$
\begin{aligned}
K=R^{-1} B^{T} P \\
u=-K x
\end{aligned}
$$

The LQR PID controller is the optimal LQR controller used to determine the gain of $\mathrm{Kp}, \mathrm{Ki}$, and $\mathrm{Kd}$ on the PID controller [13]. From the performed identification, it is known that the plant is of the order of two with the input $u(t)$, the output $y(t)$, and the transfer function of the plant:

$$
\frac{Y(s)}{U(s)}=\frac{K\left(1 \pm \frac{T_{1}}{2} s\right)}{(1+\tau s)\left(1 \pm \frac{T_{2}}{2} s\right)}=\frac{b_{1} s+b_{0}}{s^{2}+a_{1} s+a_{0}}
$$

So that the state variable for plant transfer function becomes:

$$
A=\left(\begin{array}{cc}
0 & 1 \\
-a_{0} & -a_{1}
\end{array}\right) B=\left(\begin{array}{c}
b_{1} \\
b_{0}+a_{1} b_{1}
\end{array}\right) C=(1
$$

Then, the new state is assumed, where:

$$
z=\int_{0}^{t} y d t
$$

and all states meet the condition and are available for feedback, provided $\mathrm{u} *=-\mathrm{Fx}$, so the new cost function becomes:

$$
J(u)=\int_{0}^{\infty}\left(y^{2}+p u^{2}+a z^{2}\right)
$$
by:

Now $\mathbf{J}$ as an optimization criterion that minimizes the quadratic performance index represented

$$
u^{*}(t)=-K_{p} y-K_{d} \frac{d y}{d t}-K_{i} \int_{0}^{t} y d t
$$

After the mathematical model and the constants were obtained, then designing the controller can be done by looking for the necessary controller parameters[14]. The concept of the optimum PID control method is the design of a conventional PID controller structure with tuning gain $\mathrm{Kp}, \mathrm{Ki}$, and $\mathrm{Kd}$ through LQR (Linear Quadratic Regulator) that is looking for a gain of $\mathrm{K}$ by minimizing the value of cost function. So it resulted in a control structure with a simple design, yet optimal results[15].

\section{Result and Analysis}

The system in the quadcopter is a cascade system so that the movement of corners on the quadcopter will affect the stability of the quadcopter translational motion. In this research, the test is done twice, on the movement of angle and movement of translations on the $\mathrm{x}, \mathrm{y}$, and $\mathrm{z}$-axis. 


\section{A. Movement simulation Test of Roll Angle and Pitch Angle}

The movement at the roll angle is a rolling motion on the y-axis, so the movement of the roll angle will determine the stability of the motion on the y-axis. The test is performed to determine the response of the applied controller which is expected to able to make the system output stable at the expected operating value. The drift of stable roll angle is around the 0-radian value shown in Fig 2 .

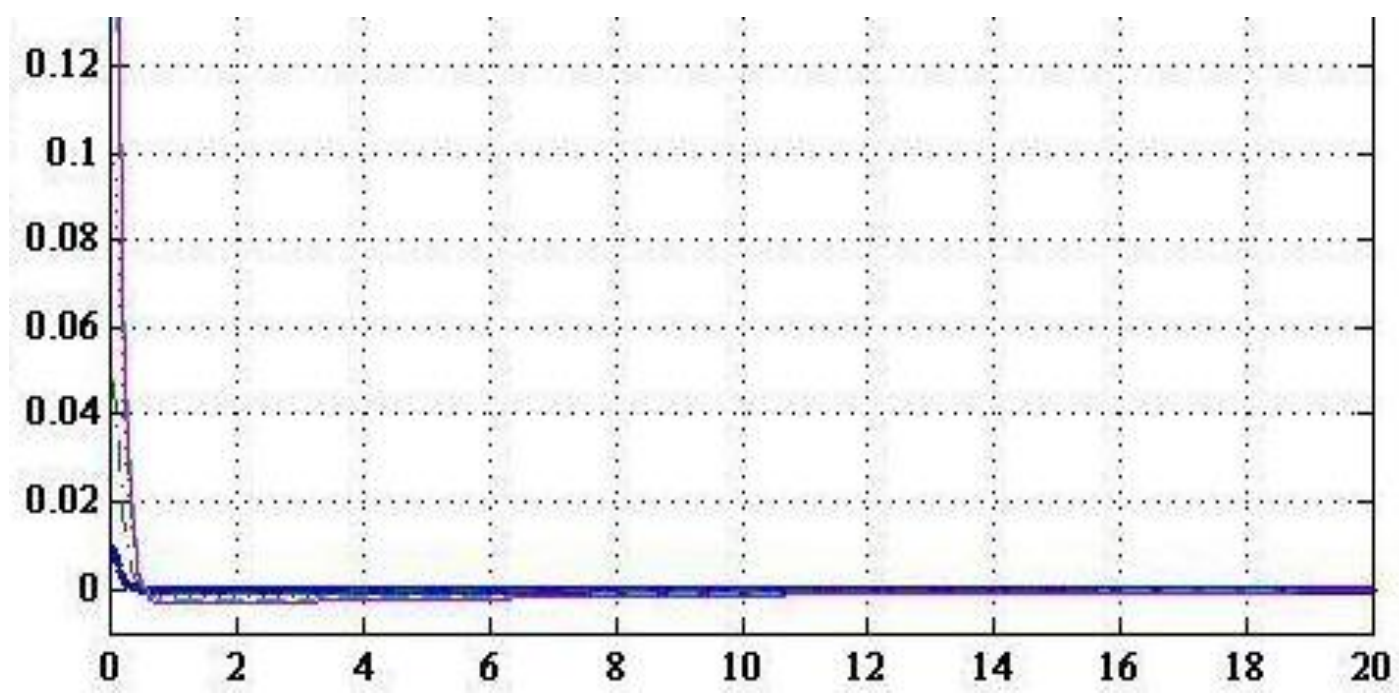

Fig. 2.The test of Roll Angle Stabilization

The results of pitch angle testing are shown in Fig 3. The results showed that the applied controller is able to control the system output response at the expected position - stable at the angle of 0 radians - although the initial conditions of the given deviation angle are varied.

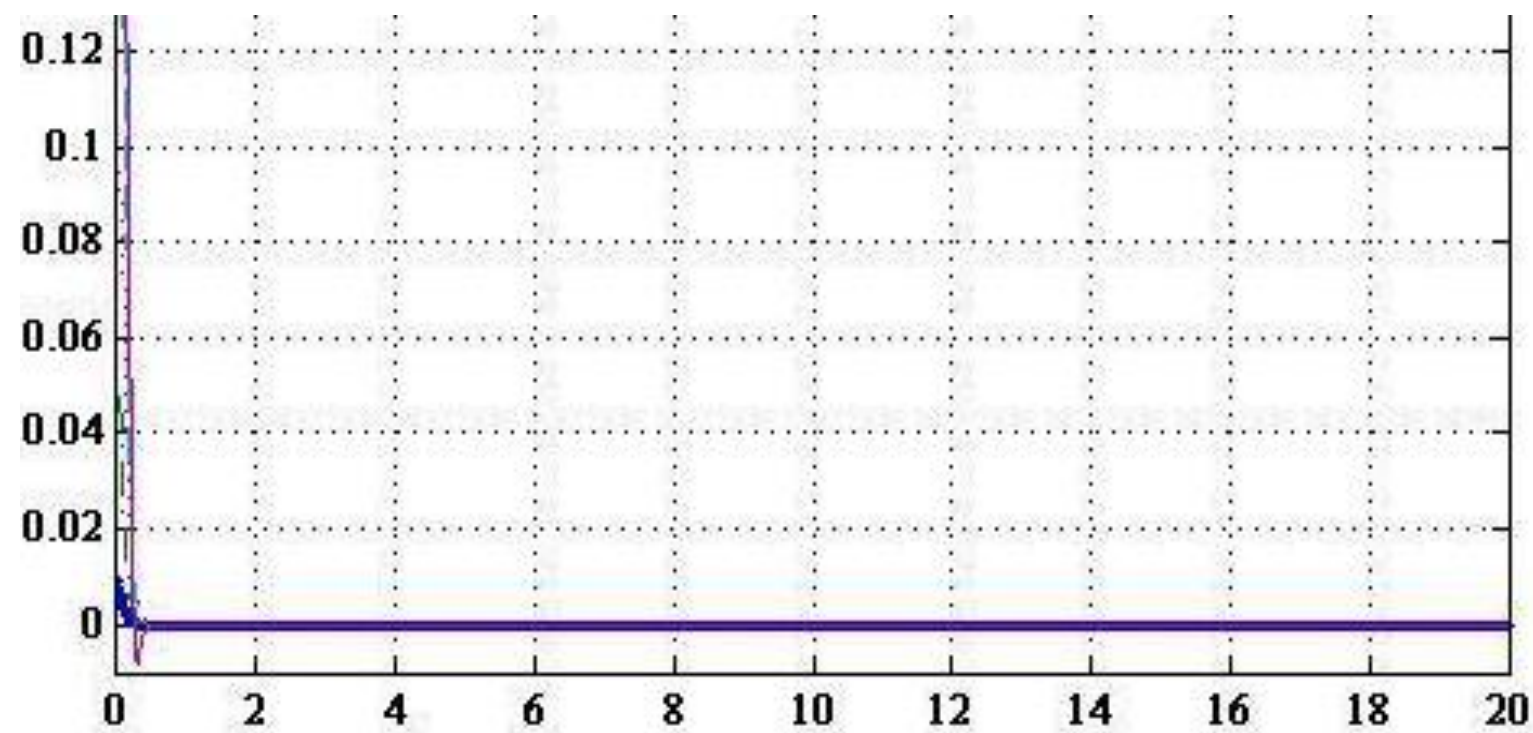

Fig. 3. The Stabilization of Pitch Angle on 0 Radian.

Fig 2 and Fig 3 showed that the applied controller for the roll and pitch angular movement is able to work optimally with the indicator of angular movement ability for stabilization at a 0 degree angle with the different initial condition. For the roll angle, the maximum time required for stabilization at 0 radians is 10 seconds and for pitch angle takes 6 seconds. The stability of the rotational motion will affect the stability of quadcopter translational motion. 
B. Quadcopter Movement Simulation on axis of $x, y$, and $z$

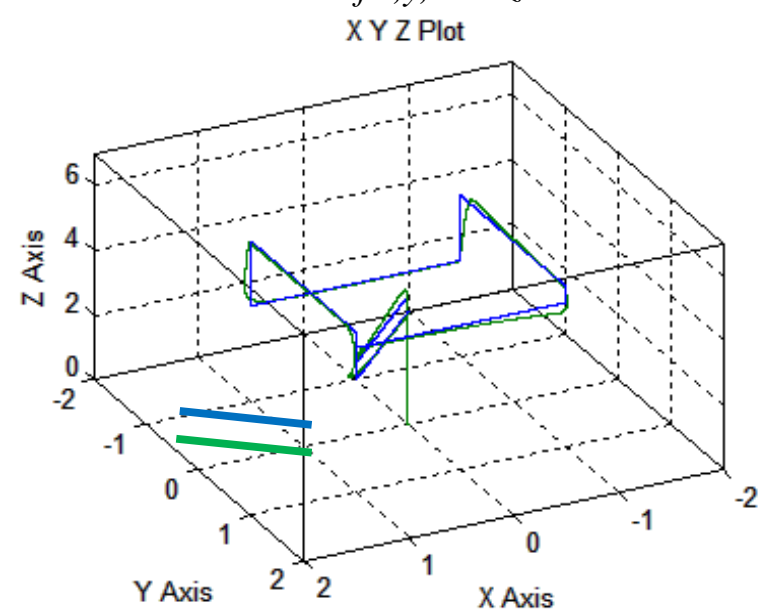

Fig. 4. Quadcopter Movement Response on the axis of $\mathrm{x}, \mathrm{y}$, and $\mathrm{z}$.

The test of translational motion is performed by observing the ability of quadcopter to follow the given reference precisely. The stability of the motion on the x-axis is determined by the stability of the pitch angle motion. While the stability of the motion on the $y$-axis is determined by the stability of the roll angle motion. As for the movement on the z-axis is based on the reference height specified by the user. Figure 4 shows that the Optimal PID controller capable of controlling the quadcopter translation movement namely the actual position quadcopter, capable of following the specified reference, with a steady state error of $4 \%$.

\section{Conclusion}

Based on the test and analysis, the conclusion as following is obtained, Optimal PID Control Method can control quadcopter rotation angle movement for angle stabilization at 0 radians position. The Optimal PID Control method can control the quadcopter translation movement to traverse the trajectory based on an optimally determined reference value with a steady state error value of less than $5 \%$

\section{References}

[1] R. Oliver, S. Y. Khoo, M. Norton, S. Adams, and A. Kouzani, "Development of a single axis tilting quadcopter," in IEEE Region 10 Annual International Conference, Proceedings/TENCON, 2017.

[2] J. J. Beaman, "Non-linear quadratic gaussian controlf,” Int. J. Control, 1984.

[3] E. Kuantama, T. Vesselenyi, S. Dzitac, and R. Tarca, "PID and Fuzzy-PID control model for quadcopter attitude with disturbance parameter," Int. J. Comput. Commun. Control, 2017.

[4] Z. Benic, P. Piljek, and D. Kotarski, "Mathematical Modelling of Unmanned Aerial Vehicles with Four Rotors," Interdiscip. Descr. Complex Syst., 2016.

[5] D. Guo, W. Yim, and K. K. Leang, "Adaptive repetitive visual-servo control of a low-flying unmanned aerial vehicle with an uncalibrated high-flying camera," in IEEE International Conference on Intelligent Robots and Systems, 2016.

[6] A. K. Das, S. Suresh, and N. Sundararajan, "A robust interval Type-2 Fuzzy Inference based BCI system," in 2016 IEEE International Conference on Systems, Man, and Cybernetics, SMC 2016 Conference Proceedings, 2017.

[7] A. Nemati and M. Kumar, "Non-linear control of tilting-quadcopter using feedback linearization based motion control," in ASME 2014 Dynamic Systems and Control Conference, DSCC 2014, 2014. 
[8] R. Syam, “Dynamics and fuzzy logic method for controlling quadcopter,” Res. J. Appl. Sci., 2016.

[9] Y. Cao and W. Ren, “Optimal linear-consensus algorithms: An lqr perspective,” IEEE Trans. Syst. Man, Cybern. Part B Cybern., 2010.

[10] M. Palanisamy, H. Modares, F. L. Lewis, and M. Aurangzeb, "Continuous-time Q-learning for infinite-horizon discounted cost linear quadratic regulator problems,” IEEE Trans. Cybern., 2015.

[11] S. L. Brunton, B. W. Brunton, J. L. Proctor, and J. N. Kutz, "Koopman invariant subspaces and finite linear representations of nonlinear dynamical systems for control," PLoS One, 2016.

[12] B. L. J. Gysen, T. P. J. Van Der Sande, J. J. H. Paulides, and E. A. Lomonova, "Efficiency of a regenerative direct-drive electromagnetic active suspension," IEEE Trans. Veh. Technol., 2011.

[13] G. K. H. S. L. Das, B. Tondu, F. Forget, J. Manhes, O. Stasse, and P. Soueres, "Controlling a multijoint arm actuated by Pneumatic muscles with quasi-DDP optimal control," in IEEE International Conference on Intelligent Robots and Systems, 2016.

[14] M. Bharatheesha, W. Caarls, W. J. Wolfslag, and M. Wisse, "Distance metric approximation for state-space RRTs using supervised learning," in IEEE International Conference on Intelligent Robots and Systems, 2014.

[15] W. An and Y. Li, "Simulation and control of a two-wheeled self-balancing robot," in 2013 IEEE International Conference on Robotics and Biomimetics, ROBIO 2013, 2013. 